\title{
The role of electron photoemission in the 'photoconductivity' of semiconducting polymers
}

\author{
Daniel Moses *, Cesare Soci, Paulo Miranda ${ }^{1}$, Alan J. Heeger \\ Institute for Polymers and Organic Solids, University of California, Santa Barbara, CA 93103, USA
}

Received 31 July 2001; in final form 26 October 2001

\begin{abstract}
We present the excitation profile of the transient and steady-state photoconductivity of poly(phenylene vinylene) and its soluble derivatives over a wide spectral range up to $h v=6.2 \mathrm{eV}$. An apparent increase in the 'photoconductivity' at $h v>3-4 \mathrm{eV}$ arises from external current generated by electron photoemission (PE). After quenching the PE by adding a gas mixture of $\mathrm{CO}_{2}+\mathrm{SF}_{6}(90 \%: 10 \%)$ into the sample chamber, the bulk photoconductivity is nearly independent of photon energy in all polymers studied. The single threshold for photoconductivity is spectrally close to the onset of $\pi-\pi^{*}$ absorption, a behavior that is inconsistent with a large exciton binding energy. (c) 2001 Elsevier Science B.V. All rights reserved.
\end{abstract}

The photophysics of semiconducting (conjugated) polymers continues to be a subject of interest and controversy [1]. The central issue is whether the lowest energy $\pi-\pi^{*}$ absorption is the result of a direct interband transition (as, for example, in GaAs) or absorption by a tightly bound exciton with the onset of the interband transition at a significantly higher energy (as, for example, in anthracene). These alternatives are intimately related to the carrier photogeneration process in this class of materials [2-4]. If charge carriers are photogenerated directly via the lowest interband

\footnotetext{
${ }^{*}$ Corresponding author. Fax: +1-805-893-4755.

E-mail address: moses@ipos.ucsb.edu (D. Moses).

${ }^{1}$ Present address: Departamento de Fisica - UNESP/Bauru, Av. Eng. Luiz Edmundo Carrijo Coube, s/n, C.P. 473, 17033360 Bauru, SP, Brazil.
}

transition, one expects to observe a single threshold for photogeneration of charged carriers close in energy to the onset of absorption. If, however, the exciton binding $\left(E_{\mathrm{b}}\right)$ energy is large, one expects to observe the threshold for photoconductivity via the lowest interband transition at an energy greater than the onset of optical absorption by $h v=E_{\mathrm{b}}$. In the latter case, typically one expects to see two thresholds for the onset of photoconductivity: the first is coincident with the onset of exciton absorption and is enabled by secondary processes, whereas the second occurs at the onset of interband absorption; i.e. at an energy much greater than the onset of exciton absorption by $E_{\mathrm{b}}$.

In addition to the very different excitation profiles outlined in the previous paragraph, the exciton model for carrier generation predicts a strong dependence of the carrier quantum efficiency $(\phi)$ on external field $(F)$ and temperature 
(T) and photon energy [5-7]. The results of experiments on semiconducting polymers are at variance with these predictions. Specifically, in conjugated polymers, $\phi$ is independent of temperature [8] and in the low to moderate field regime also independent of $F$ [9]. Moreover, recent measurements of the photocarrier density $\left(n_{\mathrm{ph}}\right)$ generated at ultrafast time scales, in zero external field, by means of transient photo-induced absorption by the infra-red active vibration (IRAV) modes, have demonstrated charge carrier photogeneration with a single threshold that is close spectrally to the onset of absorption. These results indicated that charge carriers are photogenerated with relatively high quantum efficiency; $\phi \sim 10 \%$ at $t<100 \mathrm{fs}$ in poly(phenylene vinylene), PPV, and MEH-PPV [10]. Thus, the ultrafast measurements of $n_{\mathrm{ph}}$ indicate that charge carriers (polarons) and neutral excitons are independently generated even at the earliest times [10].

Nevertheless, the carrier generation problem has remained controversial, principally because of reports of a dramatic increase in the photocurrent at energies well above the onset of absorption in PPV derivatives [11-13]. In particular, based on the action spectrum of the steady-state photoconductivity in MEH-PPV, which indicated a sharp rise above $h v \sim 3.5 \mathrm{eV}$, Chandross et al. [11] concluded that the primary excitations in this system are excitons and that the exciton binding energy is $\sim 1 \mathrm{eV}$. Such a large binding energy would imply localized Frenkel excitons and the importance of geminate recombination.

Recently, Wegewijs et al. [14] demonstrated that photoemission (PE) of electrons may contribute significantly to the photocurrent at high photon energies (photon energies approaching the work function of the semiconducting polymer). As a result, we have measured the excitation profile of the transient photoconductivity in several conjugated polymers over a wide spectral range, from the absorption edge up to $6.2 \mathrm{eV}$. We have identified and separated the distinct contribution that arises from true photoconductivity (carrier transport in the bulk of the semiconducting polymer) and from that which arises from the electron PE current. At high light intensities, the PE contribution becomes significant, particularly at photon energies above 3-4 eV. However, when this contribution is quenched by addition of $\mathrm{CO}_{2}+\mathrm{SF}_{6}$ (90\%:10\%) into the sample chamber, the bulk PC is nearly independent of photon energy over the entire spectral range up to $6.2 \mathrm{eV}$. The flat photoconductivity $\left(\sigma_{\mathrm{ph}}\right)$ excitation profile is fully consistent with the excitation profile for the photogeneration of carriers $\left(n_{\mathrm{ph}}=n_{\mathrm{e}}+n_{\mathrm{h}}=2 n_{\mathrm{e}}\right)$, as expected since $\sigma_{\mathrm{ph}}=\mathrm{e}\left(n_{\mathrm{e}} \mu_{\mathrm{e}}+n_{\mathrm{h}} \mu_{\mathrm{h}}\right)$, where $\mu_{\mathrm{e}}$ and $\mu_{\mathrm{h}}$ are the mobility of the electron and hole, respectively.

Although the number of electrons ejected from the sample surface (and possibly the Au contacts $[15,16])$ by photoemission is relatively small, the contribution of the electron PE to the transient photocurrent is significant since the fringe field from the surface contacts can induce a drift velocity that is high compared to the bulk carrier drift velocity [17]. This contribution is expected to increase at higher light intensities and higher photon energies as the probability for electron photoemission increases. As demonstrated in this Letter, although the light intensity used in steadystate photoconductivity is relatively small compared to that used in the transient PC, when the $h v$ is greater than the work function the PE contribution is significant.

The PPV samples were free standing films, tensile drawn and stretch-oriented to a draw ratio of $l / l_{0}=4$. Samples of the PPV derivatives were in the form of (nonoriented) thin films $(\sim 200 \mathrm{~nm}$ thick) that were spin cast onto alumina substrates. All films were prepared with $\mathrm{Au}$ surface contacts in the Auston switch configuration, where the gap between the electrodes (that determines the effective sample length) was approximately $200 \mu \mathrm{m}$ for the samples used for transient PC and about $20 \mu \mathrm{m}$ for the ones used for the steady-state PC.

The separation of the bulk photocurrent from that due to free electrons in vacuum was achieved by comparing the photocurrent response while the sample was kept in modest vacuum (as achieved by mechanical pump that yielded a pressure of $\sim 60$ mTorr) to that obtained with the sample in an environment of a gas mixture consisting of $90 \%$ $\mathrm{CO}_{2}$ and $10 \% \mathrm{SF}_{6}$ at atmospheric pressure. This gas mixture effectively eliminates the free electron contribution, both by impeding the electron 
motion (mostly by the $\mathrm{CO}_{2}$ molecules) $[17,18]$ and electron capture by the high electron affinity $\mathrm{SF}_{6}$ molecules [19]. Note that the residual gas pressure in the modest vacuum used in our experiments limits the magnitude of the PE contribution. In the photon energy range studied, the optical absorption due to the gas mixture is negligible [20].

The excitation source was an amplified Ti:Sapphire laser system including an optical parametric amplifier (OPA) tunable up to $\sim 4 \mathrm{eV}$, and a system that produces the third and fourth harmonics of the Ti:Sapphire laser fundamental. The laser yields pulses of $\sim 100$ fs duration at $1 \mathrm{kHz}$ rate. In order to determine the excitation profile, we used gap between the electrodes on the Auston switch of 0.2 $\mathrm{mm}$ with light intensity of $I=100 \mu \mathrm{J} / \mathrm{cm}^{2}$ perpulse, and bias field of $F=20 \mathrm{kV} / \mathrm{cm}$ that were kept constant at all photon energies. The photocurrent values were normalized to a constant photon flux of $3.5 \times 10^{14}$ photons $/ \mathrm{cm}^{2}$ per pulse, taking into account the distinct dependence of the PC on light intensity measured at each photon energy. Note that in these experiments, we used a $200 \mu \mathrm{m}$ gap rather than a $20 \mu \mathrm{m}$ gap and higher light intensities than typically used in routine transient photoconductivity measurements in our laboratory. The steady-state PC was measured by conventional modulation technique, whereby the light of a Xe lamp was dispersed by a monochromator and chopped, while the photocurrent was measured by a lock-in amplifier.

We first verified that the contribution of the PE from the underlying alumina substrates was negligible for all samples studied. In addition, we established that exposure of the samples to the gas mixture does not affect the dark conductivity, implying that the quenching of the PE by the gas mixture does not originate from chemical doping of the polymers. In the range of the bias field used of $F<25 \mathrm{kV} / \mathrm{cm}$, both the bulk photocurrent and that due to the PE were linearly proportional to $F$.

Fig. 1 shows the excitation profile of the photocurrent in MEH-PPV measured in vacuum and in an environment of $\mathrm{CO}_{2}+\mathrm{SF}_{6}(90 \%: 10 \%)$; similar results for PPV are shown in the inset. As determined from the photocurrent response obtained with the sample in the environment of the gas mixture that quenches the PE contribution, the

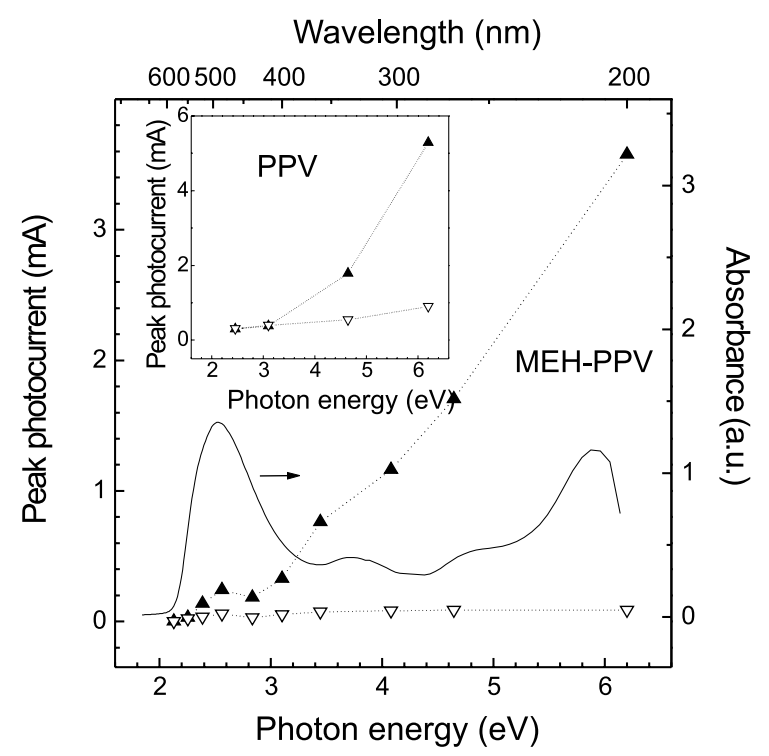

Fig. 1. Excitation profile of the peak transient PC in MEH$\mathrm{PPV}$ as measured while the sample was in vacuum $(\boldsymbol{\Delta})$ and in an environment of $\mathrm{CO}_{2}+\mathrm{SF}_{6}(90 \%: 10 \%)$ gas mixture at atmospheric pressure $(\nabla)$, normalized to a constant photon flux of $3.5 \times 10^{14}$ photons $/ \mathrm{cm}^{2}$ per pulse and $F=20 \mathrm{kV} / \mathrm{cm}$; the absorption profile of MEH-PPV is represented by the smooth line; the inset shows similar data for PPV.

bulk peak transient photoconductivity in PPV and in all the PPV derivatives studied is essentially independent of photon energy, up to $6.2 \mathrm{eV}$. As indicated in the inset of Fig. 1, the contribution of the PE to the photocurrent in PPV is negligible at photon energies below $\sim 3 \mathrm{eV}$, but becomes significant at higher photon energies and eventually dominates the photocurrent response. In contrast, MEH-PPV exhibits a PE contribution at all photon energies above the absorption edge.

Measurements on samples with a smaller gap between electrodes, $\sim 20 \mu \mathrm{m}$, using smaller light intensity and higher external field (as typically used in our transient PC measurements), yield a smaller PE contribution to the photocurrent signal than that due to the bulk PC. For example, even at a photon energy of $4.6 \mathrm{eV}$, the $\mathrm{PE}$ contribution measured in PPV sample at $I=20 \mu \mathrm{J} / \mathrm{cm}^{2} /$ pulse was a fraction $(\sim 0.5)$ of the bulk transient PC whereas at higher light intensity (used for the 0.2 mm gap) it was greater than the bulk PC by a factor of 20 (as indicated in Fig. 1). 
The independence of the bulk PC on photon energy is in disagreement with Frenkel excitons as the photoexcitations $[2,3,8,9,21]$. According to the Onsager model of geminate recombination, the higher the photon energy, the larger is the initial distance between the thermalized geminate carriers and the higher the probability of their eventual separation into mobile carriers [5-7,22]. Thus, although both $n_{\mathrm{ph}}$ and the photoconductivity are predicted by the Onsager model to increase with photon energy, both are measured to be essentially independent of photon energy.

The results in Fig. 1 are also inconsistent with the model developed by Arkhipov et al. [23,24], where the carrier generation was proposed to result from hot exciton dissociation. According to Arkhipov et al., the probability of carrier photogeneration depends on the magnitude of excess photon energy above the singlet exciton. This excess energy dissipates into the vibrational thermal

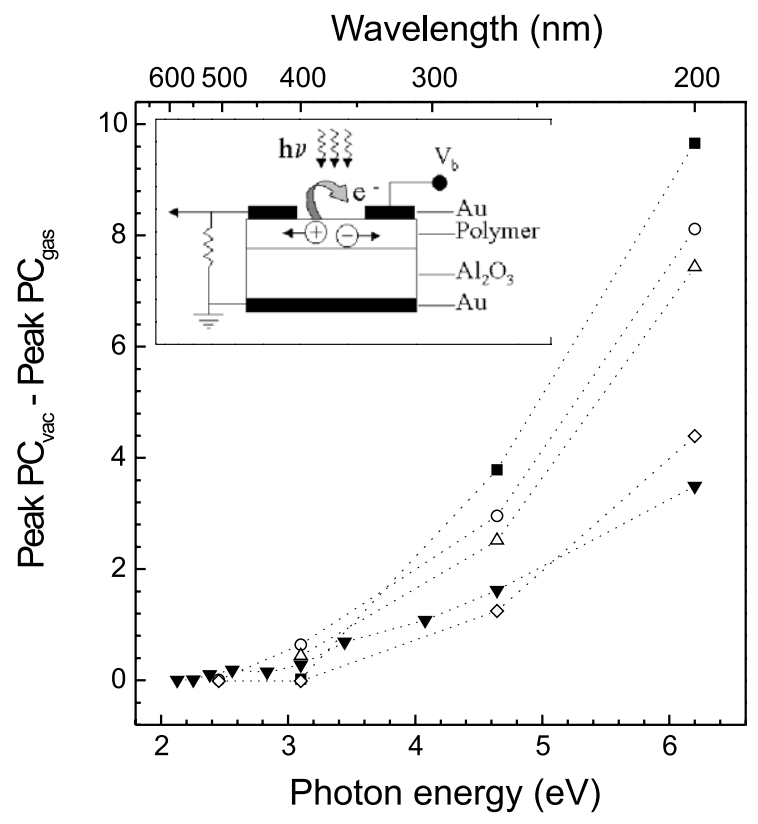

Fig. 2. Excitation profile of the PE contribution to the transport as deduced from the difference between the peak transient photocurrent measured in vacuum and that measured at atmospheric pressure of $\mathrm{CO}_{2}+\mathrm{SF}_{6}(90 \%: 10 \%)$ gas mixture in a series of PPV derivatives: (ם) BCHA-PPV, (०) BUEH-PPV, $(\triangle)$ BEH-PPV, ( $)$ MEH-PPV, $(\diamond)$ PPV; the inset shows schematically the sample measuring configuration. bath that is considered as the main source of energy required for the geminate charges to escape recombination (i.e. to escape the potential well formed by superposition of the Coulomb and external field). The observation that the bulk transient photoconductivity is independent of photon energy is inconsistent with the strong dependence on photon energy predicted by Arkhipov et al.

Fig. 2 shows the excitation profile of the PE contribution to the transient photocurrent response for all the polymers studied, as deduced from the difference between the photocurrent measured in vacuum and that measured with the sample immersed in the gas mixture. The data indicate a tendency of higher PE and smaller bulk contributions to the photocurrent in polymer derivatives with bulky side groups such as BCHAPPV and BUEH-PPV.

Fig. 3 shows the intensity dependence of the PE contribution to the photoconductive response in MEH-PPV at various photon energies. The photocurrent contribution from $\mathrm{PE}$ is proportional to $I^{n}$. The PC $\sim I^{3}$ dependence observed at $2.4 \mathrm{eV}$ indicates that the PE arises from a third-order

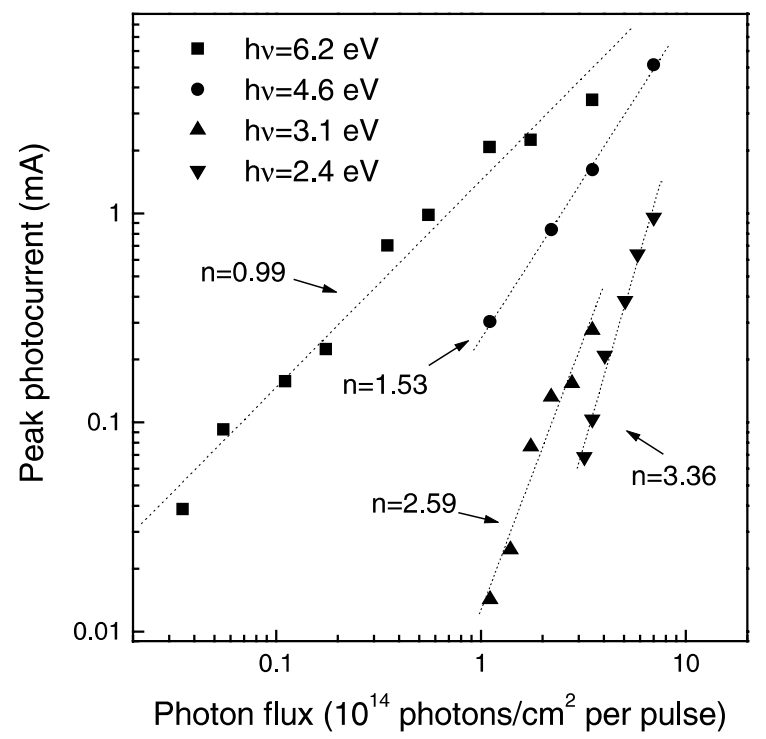

Fig. 3. The dependence of the PE contribution to the photocurrent on photon flux in MEH-PPV at various photon energies; the PE contributionis proportional to $I^{n}$; the indicated values of $n$ were obtained from fitting of the PE contribution; the different power laws are represented by the dotted lines. 
Table 1

The order of the nonlinear PE excitation process, $n$, as determined from fitting the intensity dependence of the PE contribution to the photocurrent at different photon energies, for the various PPV derivatives

\begin{tabular}{llllll}
\hline Energy $(\mathrm{eV})$ & PPV & MEH-PPV & BEH-PPV & BUEH-PPV & BCHA-PPV \\
\hline 6.2 & 1.13 & 0.99 & 1.31 & 1.12 & 1.09 \\
4.6 & 1.24 & 1.53 & 1.83 & 1.84 & 1.99 \\
3.1 & & 2.59 & 2.67 & 3.02 & \\
2.4 & & 3.36 & & & \\
\hline
\end{tabular}

process (e.g. three photon absorption), whereas at $6.2 \mathrm{eV}$, the $\mathrm{PC} \sim I$, indicating a first-order process. Thus, at $6.2 \mathrm{eV}$, the photon energy exceeds the threshold energy for photoemission, in agreement with previous analysis that concluded that the single photon PE threshold for PPV occurs at 5.2 $\mathrm{eV}$ [25]. Table 1 summarizes the values of the power law exponent, $n$, deduced for the different PPV derivatives at various photon energies.

For all the PPV derivatives, the bulk transient PC was linearly proportional to $I$ at all photon energies. The results for oriented PPV (see Figs. 1, 2 and Table 1) were deduced from measurements with light polarization perpendicular to both the chain axis and the bias field direction. At all the photon energies studied, the data obtained with light polarized perpendicular to the chain axis indicate slightly higher photoconductive response than that measured with polarized parallel to the chain axis.

Fig. 4 displays the excitation profile of the steady-state photocurrent in MEH-PPV as measured with an external field of $F=20 \mathrm{kV} / \mathrm{cm}$ while the sample was in vacuum and in an environment of $\mathrm{CO}_{2}+\mathrm{SF}_{6}(90 \%: 10 \%)$ gas mixture at atmospheric pressure, while the inset shows similar data for BEH-PPV. The data indicate a weak dependence of the bulk PC on photon energy, in agreement with the transient PC data as well as with the IRAV absorption measurements, and a rise in the apparent $\mathrm{PC}$ that results from the $\mathrm{PE}$ contribution above $\sim 4.7 \mathrm{eV}$. Although the light intensity in steady-state PC is much smaller than that used for transient PC measurements, (and thus light absorption via nonlinear processes is relatively small) the experiments indicate that for $h$ greater than the work function, the PE contribution to the steady-state photocurrent can be significant.
In summary, we have measured the excitation profile of the transient and steady-state photocurrent in various derivatives of PPV. We have detected and characterized a contribution to the transient and steady-state photocurrent due to electron photoemission. After quenching the PE contribution, the transient photoconductivity is nearly independent of the excitation energy (up to $6.2 \mathrm{eV}$ ) in all the PPV derivatives.

The photoconductivity data and the $n_{\mathrm{ph}}$ data (obtained independently from the ultrafast photoinduced IRAV mode absorption) indicate a

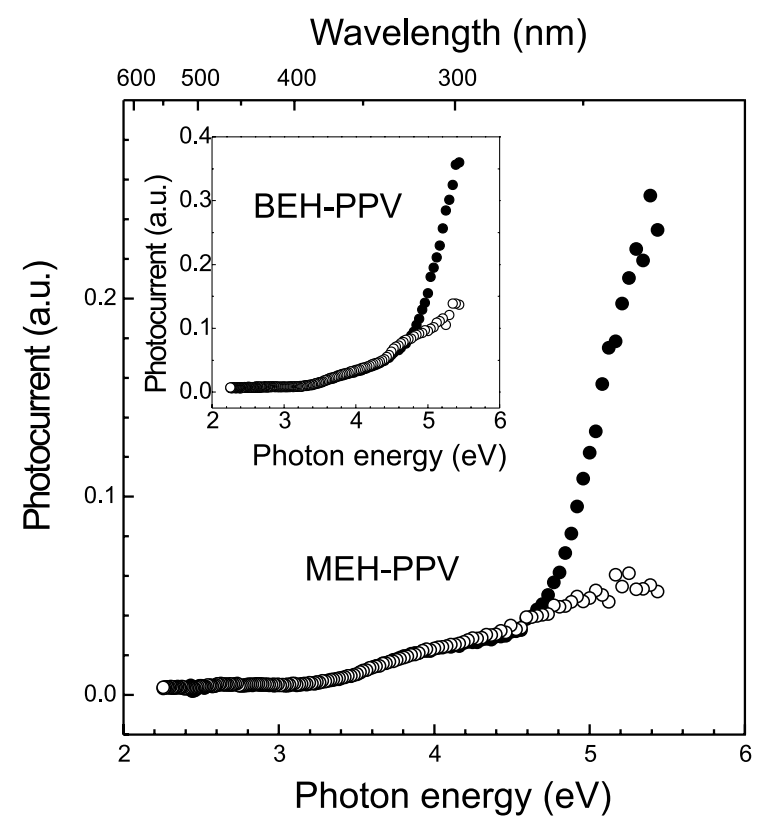

Fig. 4. Excitation profile of the steady-state photocurrent in MEH-PPV as measured while the sample was in vacuum (•) and in an environment of $\mathrm{CO}_{2}+\mathrm{SF}_{6}(90 \%: 10 \%)$ gas mixture at atmospheric pressure (o); the data were collected with an external field of $F=20 \mathrm{kV} / \mathrm{cm}$ and normalized to constant light intensity; the inset shows similar data for BEH-PPV. 
single threshold for the ultrafast photogeneration of charged carriers. Moreover, the single threshold is spectrally close to the onset of optical absorption. The absence of a second threshold in the excitation profile for the photoconductivity and $n_{\mathrm{ph}}$ implies that the lowest energy absorption results from the lowest $\pi-\pi^{*}$ interband transition and that the exciton binding energy is small. High resolution measurements of the excitation profile of the photoconductivity as a function of the electric field near the onset of interband absorption have identified the exciton in PPV through field ionization of the electron-hole bound state. In PPV, the exciton binding energy is $\approx 65 \mathrm{meV}$.

Finally, for photon energies higher than the onset of the lowest $\pi-\pi^{*}$ interband transition, carrier photogeneration in PPV and its derivatives is nearly independent of photon energy, temperature, and external field. Thus, the photophysics of semiconducting polymers is similar to that of direct gap inorganic semiconductors.

\section{Acknowledgements}

We are grateful for the useful discussions and advice we received from Dr. J. Wang in regard to the present work. The research was supported by a grant from the National Science Foundation; NSF-DMR 9812852.

\section{References}

[1] The Nature of Photoexcitations in Conjugated Polymers, World Scientific, Singapore, 1997.
[2] D. Moses, The Nature of Photoexcitations in Conjugated Polymers, World Scientific, Singapore, 1997.

[3] D. Moses, Phys. Rev. B 53 (1996) 4462.

[4] D. Moses, A.J. Heeger, in: T. Kobayashi (Ed.), Relaxation in Polymers, World Scientific, Singapore, 1993.

[5] M. Pope, C.E. Swenberg, Electronic Processes in Organic Crystals, Oxford University press, New York, 1982.

[6] R.C.E.a.G. Pfister, Photoconductivity and Related Phenomena, Elsevier, New York, 1976.

[7] H.S.a.S. Rackovsky, J. Chem. Phys. 81 (1984) 1994.

[8] D. Moses, J. Wang, G. Yu, et al., Phys. Rev. Lett. 80 (1998) 2685.

[9] D. Moses, H. Okumoto, C.H. Lee, et al., Phys. Rev. B 54 (1996) 4748.

[10] D. Moses, A. Dogariu, A.J. Heeger, Phys. Rev. B 61 (2000) 9373.

[11] M. Chandross, S. Mazumdar, S. Jeglinski, et al., Phys. Rev. B 50 (1994) 14702.

[12] A. Kohler, D.A.d. Santos, D. Beljonne, et al., Nature 392 (1998) 903.

[13] S. Bart, H. Bässler, Phys. Rev. Lett. 79 (1997) 4445.

[14] B.R. Wegewijs, G. Dicker, J. Piris, et al., Chem. Phys. Lett. $332(2000) 79$.

[15] J.P. Girardeau-Montaut, C. Girardeau-Montaut, Appl. Phys. Lett. 62 (1993) 426.

[16] N.A. Papadoggianis, S.D. Moustaizis, J. Phys. D 34 (2001) 499.

[17] Y. Itikawa, Phys. Fluids 16 (1973) 831.

[18] R.D. Hake Jr., A.V. Phelps, Phys. Rev. 158 (1967) 70.

[19] F.C. Fehsenfeld, J. Chem. Phys. 53 (1970) 2000.

[20] See: F. Bastien, P.A. Chatterton, E. Marode, et al., J. Phys. D 18 (1985) 1327, and references therein.

[21] P. Miranda, D. Moses, A.J. Heeger, Phys. Rev. B, Rapid Commun. 64 (8) (2001) 81201.

[22] L. Onsager, Phys. Rev. 54 (1938) 554.

[23] V.I. Arkhipov, E.V. Emelianova, H. Bassler, Phys. Rev. Lett. 82 (1999) 1321.

[24] V.I. Arkhipov, E.V. Emelianova, H. Bassler, Chem. Phys. Lett. 340 (2001) 517.

[25] See: W.R. Salaneck, R.H. Friend, J.L. Bredae, Phys. Rep. 319 (1999) 231, and references therein. 